\title{
Ways in which coherence is confirmation conducive
}

\author{
Luca Moretti \\ Centre for Time \\ University of Sydney \\ luca.moretti@arts.usyd.edu.au
}

(17 April 2006)

\begin{abstract}
Recent works in epistemology show that the claim that coherence is truth conducive - in the sense that, given suitable ceteris paribus conditions, more coherent sets of statements are always more probable - is dubious and possibly false. From this, it does not follows that coherence is a useless notion in epistemology and philosophy of science. Dietrich and Moretti 2005 have proposed a formal of account of how coherence is confirmation conducive - that is, of how the coherence of a set of statements facilitates the confirmation of such statements. This account is grounded in two confirmation transmission properties that are satisfied by some of the measures of coherence recently proposed in the literature. These properties explicate everyday and scientific uses of coherence. In his paper, I review the main findings of Dietrich and Moretti 2005 and define two evidence gathering properties that are satisfied by the same measures of coherence and constitute further ways in which coherence is confirmation conducive. At least one of these properties vindicates important applications of the notion of coherence in everyday life and in science.
\end{abstract}

\section{Introduction}

Intuitively, a set of statements (beliefs, hypotheses) is coherent if its members "hang together". For instance, it seems coherent to say that I am Italian and that I speak Italian, but incoherent to say that I am Italian and that I do not speak Italian. Philosophers also emphasize that, unlike the notion of logical consistency, coherence comes in degrees. Several authors have recently put forward probabilistic measures of coherence that account for both these intuitions. Coherence measures have been defined, for instance, by Shogenji 1999, Olsson 2002, Fitelson 2003 and 2004, and Bovens and Hartmann 2003. 
Some philosophers find it also intuitive that coherence is truth conducive in the sense that, given suitable and non-ad hoc ceteris paribus conditions, more coherent sets are always more probable. This thesis is however problematic, as shown by many counterexamples; it notably appears satisfied by none of the coherence measures mentioned above. ${ }^{1}$ This sense of truth conduciveness of coherence has been further challenged by general impossibility theorems presented in Bovens and Hartmann 2003 and Olsson 2005. ${ }^{2}$ From these negative results, it does not follow, however, that coherence is a useless notion in epistemology and in philosophy of science. In Dietrich and Moretti 2005, we have proposed a mathematically well-defined sense in which coherence is confirmation conducive - that is, a well definite sense in which the coherence of a set of statements facilitates the confirmation of its members. We do not focus on the probability of a set of statements but on its (incremental) confirmation. We show that, if a member of a statement set is confirmed by evidence and the set is sufficiently coherent on a suitable coherence measure, confirmation is transmitted to the conjunction of all the members of that set. We also show that, if a member of a statement set is confirmed by evidence and the set is sufficiently coherent on a suitable coherence measure, confirmation is transmitted to each member of the set. We call the first property confirmation transmission to the conjunction (CTC), and the second confirmation transmission (CT).

These two confirmation transmission properties establish an important link between Bayesian accounts of coherence and Bayesian theories of confirmation. These properties also show that coherence, if measured on a coherence measure that satisfies (CTC) or (CT), is truth conducive conditional on evidence confirming a member of a statement set. In the sense that if a member of a sufficiently coherent set of statements is confirmed by evidence, the

\footnotetext{
${ }^{1}$ For a list of papers relevant to this discussion, see Dietrich and Moretti 2005: 403, note 1.

${ }^{2}$ See also the introductory article to this special issue of Synthese.
} 
probability of the statements in the set will increase. ${ }^{3}$

Interestingly, these two confirmation transmission properties are satisfied by some but not all coherence measures. For instance, (CTC) and (CT) are both satisfied by Olsson's measure, while Fitelson's satisfies only a weaker version of (CT), and Shogenji's neither. ${ }^{4}$ Although these properties appear intuitive (in the sense of reflecting features of our pretheoretical notion of coherence) and explicate daily life inferences and confirmation methods in science, we however do not claim that instantiating confirmation transmission properties is an essential feature of any coherence measure. As coherence measures may serve purposes different than transmitting confirmation.

In this paper, after reviewing the main findings of Dietrich and Moretti 2005, I define two evidence gathering properties that account for further ways in which coherence is confirmation conducive. The first property is to the effect that, if an evidential statement confirms a hypothesis and the evidential statement is sufficiently coherent with other evidential statements, then the conjunction of all these statements also confirms the hypothesis. The second property is such that, if an evidential statement confirms a hypothesis and the evidential statement is sufficiently coherent with other evidential statements, then each of these statements also confirms the hypothesis. I call the first property conjunctive evidence gathering (CEG), and the second property evidence gathering (EG). (CEG) is formally equivalent to (CTC), and (EG) is formally equivalent to (CT). Consequently, a

\footnotetext{
${ }^{3}$ In Dietrich and Moretti 2005: 404, we interpret the confirmation transmission properties as ways in which coherence is truth conducive in this conditional sense rather than as ways in which coherence is confirmation conducive.

${ }^{4}$ In Dietrich and Moretti 2005: 423, we give a proof that Shogenji's measure does not satisfy (CT) and a sketched proof that Fitelson's measure does not satisfy the same propriety. It may appear puzzling that the coherence measure proposed by Shogenji, justly celebrated for its other appealing properties, fails to justify our confirmation transmission properties. We however recognize that Shogenji's measure satisfies a simple but important property of confirmation transmission based on a screening off relation. See: 415-17.
} 
coherence measure satisfies (CEG) if and only if satisfies (CTC), and a coherence measure satisfies (EG) if and only if satisfies (CT). ${ }^{5}$ I argue that (CEG) vindicates applications of the notion of coherence in everyday life and in science, and - importantly - the use of coherence to make confirmation process comply with the total evidence condition.

In particular, Sect 2 introduces the coherence measures that satisfy confirmation transmission properties. Sect 3 reviews the findings of Dietrich and Moretti 2005. Sect. 4 introduces and discusses the evidence gathering properties. Sect 5 contains the conclusions of the paper.

\section{Olsson's and Fitelson's measures of coherence}

A coherence measure is roughly a function that gives each set of statements a number that represents its degree of coherence. More exactly, consider a standard language of propositional logic and a finitely additive Kolmogorov probability distribution $P$ defined on this language. $P$ is interpreted subjectively: if $H$ is a formula of the formal language, $P(H)$ gives the degree of confidence of a rational agent in $H$. A formula $E$ is said to confirm (incrementally) another formula $H$ if and only if $P(E)>0$ and $P(H \mid E)>P(H)$, where $P(H \mid E)$ measures a rational agent's confidence in $H$ on the supposition that $E$ is true. ${ }^{6}$ A coherence measure is a function $C$ that maps every finite and non-empty set $S$ of formulae with positive probability to a real number $C(S)$. Let $\mathbf{S}$ be the set of all finite and non-empty set $S$ of formulae with positive probability.

At least two interesting coherence measures satisfy confirmation transmission

\footnotetext{
${ }^{5}$ As the evidence gathering properties are logically equivalent to the confirmation transmission properties, it is perhaps more appropriate to consider the former as different applications of the latter rather than new and distinct properties.

${ }^{6}$ For further assumptions concerning $P$, see Dietrich and Moretti 2005: 405-6.
} 
properties: Olsson 2002's and Fitelson's $2004 .^{7}$ Olsson proposes to define the coherence of a set $S \in \mathbf{S}$ as:

$$
C_{O}(S):=\frac{P\left(\bigwedge_{H \in S} H\right)}{P\left(\mathrm{~V}_{H \in S} H\right)}
$$

This measure ranges over the interval $[0,1]$. Olsson conceives of the coherence of $S$ as an agreement among the statements in $S$. The maximal agreement occurs when $S$ contains only pairwise equivalent statements; this is reflected by $C_{o}$, as, in this case, $C_{o}(S)=1$. The agreement is minimal when $S$ is logically inconsistent; accordingly, in this case, $C_{o}(\mathrm{~S})=0$. Although these two features of $C_{o}$ may appear intuitive, this measure also has aspects that may appear counterintuitive. For instance, on $C_{o}$, it turns out that the coherence of two positively dependent statements can be lower than that of two negatively dependent statements. $^{8}$

Fitelson's coherence measure aims to reflect the level of mutual support (or confirmation) of the members of a set. Fitelson defines the coherence of a set $S \in \mathbf{S}$ as the average of the degree to which the conjunction of the formulae of each non-empty subset of $S$ is supported, on a specific confirmation measure, by the conjunction of the formulae of each of the non-empty subsets of $S$ disjunct from the former subset. To put it formally:

$$
C_{F}(S):=\frac{1}{|R|} \sum_{\left(S_{1}, S_{2}\right) \in R} F\left(\Lambda_{H_{1} \in S_{1}} H_{1}, \Lambda_{H_{2} \in S_{2}} H_{2}\right) \text {. }
$$

Where $R$ is the set of all pairs $\left(S_{1}, S_{2}\right)$ of non-empty subset $S_{1}, S_{2} \subseteq S$, with $S_{1} \cap S_{2}=\varnothing$. $F$ is the Kemeny and Oppenheim 1952 measure of factual support, which is defined as follows:

$$
F(H, K):=\frac{P(K \mid H)-P(K \mid \neg H)}{P(K \mid H)+P(K \mid \neg H)}
$$

\footnotetext{
${ }^{7}$ Fitelson 2004 has presented a coherence measure that revises the one defined in Fitelson 2003.

${ }^{8}$ For further criticism, see Dietrich and Moretti 2000: 407.
} 
interpreted as -1 if $P(H)=0$ or $P(K)=0$, and as 1 if $P(\neg H)=0$ and $P(K)>0 .{ }^{9}$ For example, if $S=\left\{H_{1}, H_{2}\right\}, R=\left\{\left(\left\{H_{1}\right\},\left\{H_{2}\right\}\right),\left(\left\{H_{2}\right\},\left\{H_{1}\right\}\right)\right\}$. Consequently:

$$
C_{F}(S)=1 / 2\left[F\left(H_{1}, H_{2}\right)+F\left(H_{2}, H_{1}\right)\right] .
$$

Fitelson's measure ranges over the interval $[-1,1]$. If $S$ consists of pairwise inconsistent formulae, $C_{F}(S)=-1$ (minimal coherence). If $S$ consists of probabilistic independent formulae, $C_{F}(S)=0$. If $S$ consists of pairwise equivalent formulae, $C_{F}(S)=1$ (maximal coherence). Although these three features of $C_{F}$ may appear intuitive, this measure also possesses aspects that could be argued to be counterintuitive. For instance, on $C_{F}$, logically inconsistent sets may turn out to be more coherent than logically consistent sets. ${ }^{10}$

\section{Confirmation transmission properties}

There are cases in which evidence does confirm each member of a set of statements without confirming their conjunction. In Dietrich and Moretti 2005: 409, we give the following example: there are two particle sources such that each of them can emit either an electron or a positron or no particle at all. Annihilation obtains just in case each source emits a particle and the two particles are of opposite charge (i.e. an electron and a positron). Annihilation is observable as two photons are emanated. Let $H$ be the hypothesis that the first source emits an electron, let $H^{*}$ be the hypothesis that the second source emits an electron, and let $E$ be the observation report that annihilation occurs. Although $E$ does confirm both $H$ and $H^{*}$ separately, $E$ cannot but disconfirm their conjunction $H \wedge H^{*}$.

Cases in which evidence confirms each member of a set of hypotheses without confirming their conjunction can also be found in everyday life. Consider the following: Ruth has two buddies, Carla and Rebecca. Some time ago, Carla and Rebecca had a ruinous

\footnotetext{
${ }^{9}$ This is indeed a generalization of Fitelson 2004's coherence measure: while Fitelson's measure applies only if $P$ is regular, this condition is not required here.

${ }^{10}$ For further criticism, see Bovens and Hartmann 2003: 51-3.
} 
argument, and now they dislike so intensely one another that can hardly stay in the same room at the same time. Ruth, Carla and Rebecca like to party at John's studio from time to time, ${ }^{11}$ and at John's studio there is a party just now. $H$ is your hypothesis that Carla is at that party. $H^{*}$ is your hypothesis that Rebecca is at that party. $E$ is your evidence that Ruth is there (e.g. you are told it). Since Carla and Rebecca are both close friends of Ruth, $E$ increases your confidence in both $H$ and $H^{*}$ individually taken. So, $E$ confirms each of them separately. Yet $E$ does not confirm $H \wedge H^{*}$. As you know that Carla (Rebecca) would hardly remain in the same room where Rebecca (Carla) is, even if her best friend is there.

It may be suggested that one of the reasons why $E$ does not confirm the conjunction $H$ $\wedge H^{*}$, in the above example, is that, given background knowledge, the two conjuncts $H$ and $H^{*}$ do not cohere sufficiently. In other words, the impression could be that if $H$ and $H^{*}$ had cohered sufficiently (for instance, given different background information), $E$ would have confirmed their conjunction. The property of confirmation transmission to the conjunction stems from the attempt to generalize and make intuitions of this type formally precise.

\section{Confirmation Transmission to the Conjunction (CTC). For any formulae $E$ and $H$} such that $E$ confirms $H$, there exist a (non-trivial) ${ }^{12}$ coherence threshold $c=c_{E, H} \in \mathbf{R}$ such that, for any set $S \in \mathbf{S}$ containing $H$ with coherence $C(S) \geq c\left(\right.$ and $P\left(\wedge_{H^{*} \in S} H^{*}\right)>$ $0), E$ confirms the conjunction $\wedge_{H^{*} \in S} H^{*}{ }^{13}$

\footnotetext{
${ }^{11}$ Of course, not all three at the same time!

${ }^{12}$ By 'non-trivial', we mean that $c<\sup _{S \in \mathbf{S} \text { s.t. } H \in S} C(S)$. Such supremum equals to the maximum coherence level if $C$ assigns maximal coherence to any set $S \in \mathbf{S}$ of pairwise equivalent formulae. This is the case with both Olsson's and Fitelson's coherence measure.

${ }^{13}$ (CTC) and the other properties of confirmation conduciveness analysed in this paper can be reformulated in terms of properties of a coherence ordering $\geq$ on $\mathbf{S}$, where a coherence ordering - to comply with Bovens and Hartmann 2003's impossibility theorem - may be incomplete. (See Dietrich and Moretti 2005: 409, footnote 7).
} 
We have proven that $C_{\mathrm{o}}$ satisfies (CTC) with coherence threshold $c_{E, H}=1 /[1+P(E \mid H)-P(E)]$ (cf.: 410). This threshold behaves in accordance with our expectations: it is the higher, the less dependent $E$ and $H$ are in the sense that $P(E \mid H)-P(E)$ is smaller. Besides, $c_{E, H}$ tends to 1 (maximal coherence) as $P(E \mid H)-P(E)$ tends to 0 (notice that $E$ confirms $H$ if and only if $P(E \mid H)-P(E)>0)$. It should be clear that exceeding this threshold constitutes a sufficient but not necessary condition for the confirmation of the conjunction $\bigwedge_{H^{*} \in S} H^{*}$. Consider for instance a set $S \in \mathbf{S}$ containing a formula $H$ confirmed by a formula $E$. Suppose that $P\left(\Lambda_{H^{*} \in S}\right.$ $\left.H^{*}\right)>0$ but that $C_{\mathrm{o}}(S)<c_{E, H}$. If $H$ entails $E$, the conjunction $\wedge_{H^{*} \in S} H^{*}$ entails $E$ too. As a result, this conjunction must be confirmed by $E$.

Consider again Ruth and her friends Carla and Rebecca. Let us suppose that, now, Carla and Rebecca no longer dislike one another but are very good friends and usually spend time together. $H$ is your hypothesis that Carla is at John's party. $H^{*}$ is your hypothesis that Rebecca is at John's party. $E$ is your evidence that Ruth is there. $E$ confirms both $H$ and $H^{*}$ individually. Does $E$ confirm the conjunction $H \wedge H^{*}$ ? The answer appears positive, as now $H$ and $H^{*}$ "hang together" given background knowledge. Assume therefore that $\left\{H, H^{*}\right\} \in$ $\mathbf{S}$, that the coherence of $\left\{H, H^{*}\right\}$ is measured on a function $C$ that satisfies (CTC), and that the probability distribution $P$, which reflects background information about Ruth, Carla and Rebecca, is such that $P\left(H \wedge H^{*}\right)>0$ and $C\left(\left\{H, H^{*}\right\}\right) \geq c_{E, H}$ or $c_{E, H^{*}}$. A formal explication of this intuitive confirmation process is at hand now, as it just follows that $E$ must confirm $H \wedge$ $H^{*}$.

In science, (CTC) provides a formal rationale for the method of confirming a theory by confirming its parts (where a theory is simply a conjunction of hypotheses) (cf.: 410-11). Let us consider a hypothetical application of this method in biology. $S$ is a low-level descriptive theory about wasps that consists of only three hypotheses: $H, H_{1}$ and $H_{2} . H$ says that the wasps of species $A$ have digestive system of type $B . H_{1}$ says that the wasps of species 
$A$ have nervous system of type $B_{1} . H_{2}$ says that the wasps of this species have exoskeleton of type $B_{2}$. Suppose furthermore that evidence $E$ consists of a significant number of caterpillars of a certain kind regularly observed in regions inhabited by the wasps of species $A$, but rarely observed elsewhere. Assume, finally, that the digestion of the blood of such caterpillars just requires a digestive system of type $B$. E plausibly confirms $H$ (given background information that wasps of many species eat caterpillars), but it may be unclear whether $E$ confirms the conjunction $H \wedge H_{1} \wedge H_{2}$, considering that $H$ does not entail $E$. Can we assert that, given that a part of $S$ is confirmed, $S$ as a whole is confirmed? A quite natural way to answer this question is to evaluate whether the parts of $S$ "hang together" sufficiently, so that the confirmation of one part can be communicated to the whole. If coherence is measured on a function $C$ that satisfies (CTC), this intuitive procedure finds a formal vindication: suppose that $S \in \mathbf{S}$ and that the probability function $P$ is such that $P\left(H \wedge H_{1} \wedge H_{2}\right)>0$ and $C(S) \geq c_{E, H}$ (where $P$ reflects background information about wasps). In this case, $E$ must necessarily confirm the conjunction $H \wedge H_{1} \wedge H_{2}$.

Let us turn to the second confirmation transmission property identified in Dietrich and Moretti 2005. Consider the following situation: you know that Rebecca has a boyfriend but you know (nearly) nothing about him. One day, you find out that his name is Takashi. From this, you infer two things: that he is likely to speak Japanese and that he has probably almondshaped eyes. Your reasoning can be described like so: evidence $E$ that Takashi is the name of Rebecca's boyfriend, given your background information about Japanese people, raises your confidence in the hypothesis $H$ that Rebecca's boyfriend is Japanese. Considering that $H$, the hypothesis $H_{1}$ that Takashi speaks Japanese and the hypothesis $H_{2}$ that Takashi has almondshaped eyes "hang together" very well (given your background knowledge), evidence $E$ raises your confidence also in $H_{1}$ and $H_{2}$ individually taken. The property of coherence that apparently underlies inferences of this kind is to the effect that, if a member of a statement set 
is confirmed and the set is sufficiently coherent, confirmation is transmitted to each member of the set.

Confirmation Transmission (CT). For any formulae $E$ and $H$ such that $E$ confirms $H$, there exist a (non-trivial) ${ }^{14}$ coherence threshold $c=c_{E, H} \in \mathbf{R}$ such that, for any set $S \in$ S containing $H$ with coherence $C(S) \geq c, E$ confirms each member of $S .^{15}$

It is useful to define a weaker version of this property.

Weak Confirmation Transmission (CT*). For any formulae $E$ and $H$ such that $E$ confirms $H$, there exist a (non-trivial) ${ }^{16}$ coherence threshold $c=c_{E, H} \in \mathbf{R}$ such that, for any binary set $S^{*} \in \mathbf{S}$ containing $H$ with coherence $C(S) \geq c, E$ confirms both members of $S^{*}$.

$C_{\mathrm{o}}$ satisfies $(\mathrm{CT})$ - and so $\left(\mathrm{CT}^{*}\right)$ - with, once more, coherence threshold $c_{E, H}=1 /[1+P(E \mid H)$ $-P(E)]$ (cf.: 413 and 418-21). Furthermore, $C_{\mathrm{F}}$ satisfies $\left(\mathrm{CT}^{*}\right)$ with coherence threshold $c_{E, H}=$

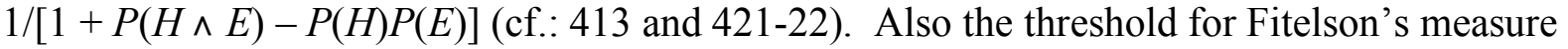
has intuitive and desirable features: it is the higher, the less dependent $E$ and $H$ are in the sense that $P(H \wedge E)-P(H) P(E)$ is smaller. Moreover, $c_{E, H}$ tends to 1 (maximal coherence) as $P(H \wedge E)-P(H) P(E)$ tends to 0 (notice that $E$ confirms $H$ if and only if $P(E \wedge H)-P(E) P(H)$ $>0$ ). We conjecture that Fitelson's measure (and other interesting coherence measures yet to

\footnotetext{
${ }^{14}$ See note 12 above.

${ }^{15}$ The reader might wonder what relations hold between the (CTC) thresholds and the (CT) thresholds. In Dietrich and Moretti (2005: 410) we single out one relation: any coherence measure $C$ that satisfies (CT) and $C\left(S \cup\left\{\wedge_{H \in S} H\right\}\right) \geq C(S)$ for every $S \in \mathbf{S}$ with $P\left(\wedge_{H \in S} H\right)>0$, also satisfies (CTC), and each possible coherence threshold $c_{E, H}$ in (CT) will be a possible coherence threshold $c_{E, H}$ in (CTC). Olsson's is one of these coherence measures.

${ }^{16}$ See note 12 above.
} 
be specified) might also satisfy conditions that come very close to (CT) and (CTC), obtained by allowing the coherence threshold to depend on the size of the set $S$ (cf.: 412 ).

The formal explication of the intuitive reasoning about Takashi says that, if the coherence of the set $\left\{H, H_{1}, H_{2}\right\} \in \mathbf{S}$ is measured on a coherence function $C$ that satisfies (CT) and assuming that the probability distribution $P$, which reflects background information about Japanese people, is such that $C\left(\left\{H, H_{1}, H_{2}\right\}\right) \geq c_{E, H}, E$ must confirm both $H_{1}$ and $H_{2}$. $\left(\mathrm{CT}^{*}\right)$ provides a formal explication of the method of indirect confirmation used in science; namely, the practice to consider a hypothesis $H^{*}$ confirmed by evidence $E$ if $E$ confirms another hypothesis $H$ related to $H^{*}$ (see, for instance, Laudan and Leplin 1991). This method applies in cases in which it is not immediately obvious that $E$ confirms $H^{*}$, while it is apparent that $E$ confirms $H$. By this procedure, we do not confirm a theory (i.e. a conjunction of hypotheses) by confirming its parts but, rather, we confirm a hypothesis by confirming another related hypothesis. A crucial question is what it means, here, that two hypotheses $H$ and $H^{*}$ are related. Our suggestion is that 'related' could be interpreted as 'sufficiently coherent' (cf. Dietrich and Moretti 2005: 412).

Consider the following historical case from physics: ${ }^{17}$ the general theory of relativity entails the statement $H$ that gravity will bend the path of a light ray if the ray passes close to a massive body. Scientists first confirmed $H$ during a total eclipse of the sun in 1919. Since then, $H$ has been repeatedly confirmed by more and more accurate tests. Although the general theory of relativity also entails the hypothesis $H^{*}$ that there are gravitational waves, physicists gave some consideration to it only in the early 1960s, and the first experiments to test $H^{*}$ were attempted just in the late1960s. One possible interpretation of the scientific

\footnotetext{
${ }^{17}$ In Dietrich and Moretti 2005: 414-15, we analyse a historical case also described in Laudan and Leplin 1991: the indirect support for the climate change hypothesis via confirmation of the continental drift hypothesis.
} 
community's behaviour is that only when evidence $E$ supporting $H^{18}$ became massive, the same evidence was perceived as indirectly confirming $H^{*} . H^{*}$ was then taken seriously and became sufficiently believable to undergo more specific tests. Since $H$ and $H^{*}$ are both deducible from the general theory of relativity, an intuitive explanation of the fact that $E$ appears to confirm $H^{*}$ to some degree is that Einstein's theory, by its unifying power, makes $H$ and $H^{*}$ cohere. The result is that confirmatory boost for the first, if sufficiently strong, can be transmitted to the second. ${ }^{19}$

When coherence is measured on a measure $C$ that satisfies $\left(\mathrm{CT}^{*}\right)$, this intuitive procedure has a formal explication. Suppose that $\left\{H, H^{*}\right\} \in \mathbf{S}$ and that the probability function $P$, which reflects background information, is such that $C\left(\left\{H \wedge H^{*}\right\}\right) \geq c_{E, H}$ (where background information says, among other things, that both $H$ and $H^{*}$ are deducible from Einstein's theory). It follows from this that $E$ must confirm $H^{*}$. Notice that I have suggested that $E$ ended up confirming $H^{*}$ only when the confirmation of $H$ given $E$ became sufficiently powerful. The focus here is on the strength of (direct) confirmation rather than on the strength of coherence. But this makes perfectly sense in our model. For the threshold $c_{E, H}$ typically decreases as $E$ and $H$ become more dependent (this happens with both Olsson's and Fitelson's measures), with the consequence that a smaller degree of coherence between $H$ and $H^{*}$ already suffices for confirmation transmission.

\footnotetext{
${ }^{18} H$ could perhaps be conjoined with the hypothesis of the gravitational redshift of light and with the description of Mercury's perihelion. Both these hypotheses follow from Einstein's theory and were accurately confirmed by observations before the late 1960's.

${ }^{19}$ Notice that the simple fact that $H$ and $H^{*}$ are both deducible from the same statement cannot suffice for the transmission of confirmation from one to the other, as any two arbitrary statements are always deducible from their logical conjunction. If this were sufficient for the transmission of confirmation, the absurdity that anything confirms anything would quickly follow. Consider any statement $A$. $A$ confirms itself. $A$ is entailed by $A \wedge B$, where $B$ is any statement. $B$ is entailed by $A \wedge B$. $A$ thus confirms $B$. Cf. Okasha 1997.
} 
It is worth emphasizing that if coherence is confirmation conducive, coherence is also disconfirmation conducive. (A formula $E$ is said to disconfirm (incrementally) another formula $H$ if and only if $P(E)>0$ and $P(H \mid E)<P(H)$ ). Accordingly, (CTC) and (CT) can be reformulated in term of disconfirmation (and the same holds true of (CEG) and (EG), which I will define in the next section). For instance, a coherence measure satisfies (CTC) if and only if satisfies the following condition:

Disconfirmation Transmission to the Conjunction (DTC). For any formulae $E^{\prime}$ and $H$ such that $E^{\prime}$ disconfirms $H$, there exist a (non-trivial) ${ }^{20}$ coherence threshold $c=c_{E^{\prime}, H} \in$ $\mathbf{R}$ such that, for any set $S \in \mathbf{S}$ containing $H$ with coherence $C(S) \geq c$ (and $P\left(\wedge_{H^{*} \in S} H^{*}\right)$ $>0), E^{\prime}$ disconfirms the conjunction $\wedge_{H^{*} \in S} H^{*}$.

This is true because, for any three formulae $E, E^{\prime}$ and $H$, such that $E^{\prime}$ is the logical negation of $E, E$ confirms $H$ if and only if $E^{\prime}$ disconfirms $H$. Moreover, as $P(E \mid H)=1-P\left(E^{\prime} \mid H\right)$ and $P(E)=1-P\left(E^{\prime}\right), C_{\mathrm{o}}$ satisfies (DTC) with coherence threshold $c_{E^{\prime}, H}=1 /\left[1+P\left(E^{\prime}\right)-P\left(E^{\prime} \mid H\right)\right]$.

\section{Evidence gathering properties}

The evidence gathering properties are definable by exchanging the roles of $E$ and $H$ in (CTC) and (CT); the result is that $S$ is no longer a set of hypotheses but becomes a set of evidential statements.

Conjunctive Evidence Gathering (CEG). For any formulae $E$ and $H$ such that $E$ confirms $H$, there exist a (non-trivial) ${ }^{21}$ coherence threshold $c=c_{E, H} \in \mathbf{R}$ such that, for any set $S \in \mathbf{S}$ containing $E$ with coherence $C(S) \geq c\left(\right.$ and $\left.P\left(\Lambda_{E^{*} \in S} E^{*}\right)>0\right)$, the conjunction $\wedge_{E^{*} \in S} E^{*}$ confirms $H$.

\footnotetext{
${ }^{20}$ See note 12 above.

${ }^{21}$ By 'non-trivial', I mean, again, that $c<\sup _{S \in \mathbf{S} \text { s .t. } E \in S} C(S)$.
} 
Evidence Gathering (EG). For any formulae $E$ and $H$ such that $E$ confirms $H$, there exist a (non-trivial) ${ }^{22}$ coherence threshold $c=c_{E, H} \in \mathbf{R}$ such that, for any set $S \in \mathbf{S}$ containing $E$ with coherence $C(S) \geq c$, each member of $S$ confirms $H$.

In analogy with $\left(\mathrm{CT}^{*}\right),\left(\mathrm{EG}^{*}\right)$ can be defined as a version of $(\mathrm{EG})$ restricted to binary sets $S$ only.

As, for any two formulae $E$ and $H, E$ confirms $H$ if and only if $H$ confirms $E$, and considering that (CEG) is defined by exchanging the roles of $E$ and $H$ in (CTC), it follows that a coherence measure satisfies (CEG) if and only satisfies (CTC). In the same way, a coherence measure satisfies (EG) if and only if satisfies (CT). Accordingly, $C_{\mathrm{o}}$ satisfies both (CEG) and (EG). $C_{\mathrm{o}}$ satisfies both of them with the same coherence threshold as in (CTC) and (CT) but with the roles of $E$ and $H$ exchanged; so with $c_{E, H}=1 /[1+P(H \mid E)-P(H)]$. Notice that the difference $P(H \mid E)-P(H)$ just gives the degree of confirmation of $H$ by $E$ on the confirmation measure $d$, one of the most popular in the Bayesian literature. This threshold appears very natural: it is the higher, the less $E$ confirms $H$. Besides, $c_{E, H}$ tends to 1 (maximal coherence) as the confirmation $H$ given $E$ tends to $0 . C_{\mathrm{F}}$ satisfies the weaker version (EG*) of (EG) with coherence threshold $c_{E, H}=1 /[1+P(H \wedge E)-P(H) P(E)]$. While (EG) and (EG*) can perhaps explain interesting uses of coherence in everyday life and in science, ${ }^{23}$ (CEG) actually provides formal explication of important applications of coherence in both areas. In the remainder of the section, I will focus on some of these applications and their formal explication.

\footnotetext{
${ }^{22}$ See note 21 above.

${ }^{23}$ Consider, for instance, this possible use of coherence explicable in terms of (EG): initially, it is not evident to me that my actual evidence $E^{*}$ confirms hypothesis $H$. I then realize that $E^{*}$ is highly coherent with a possible evidence $E$ that confirms $H$. I thus infer that $E^{*}$ does confirm $H$.
} 
Uses of coherence can help ascertain whether a conjunction of evidential statements confirms or does not confirm a hypothesis. Often, answering a question of this type is not trivial. For instance, if certain evidential statements confirm, separately, a given hypothesis, the conjunction of these statements need in general not confirm that hypothesis. Consider a variation of the example about particle sources discussed above: there are two particle sources such that each of them can emit either an electron or a positron or no particle at all. Annihilation obtains just in case each source emits a particle and the two particles are of opposite charge. $E$ is now the observation that the first source emits an electron, $E^{*}$ is the observation that the second source emits an electron, and $H$ is the hypothesis that annihilation occurs. Although both $E$ and $E^{*}$ individually confirm $H$, their conjunction $E \wedge E^{*}$ disconfirms $H{ }^{24}$

Consider an example from a different area: a detective investigates whether $\mathrm{Mr} \mathrm{X}-\mathrm{a}$ murderer active in Sydney - is from continental Europe. Let $H$ be this hypothesis. Preliminary investigation places $H$ among initially equally plausible alternatives (e.g. $\mathrm{Mr} \mathrm{X}$ may also be African or American or Australian or non-continental European). The detective receives in succession four reports from reliable witnesses who heard $\mathrm{Mr}$ X speak English in Sydney on different occasions. Report $E$ says that $\mathrm{Mr} \mathrm{X}$ spoke on the phone with a pronounced Spanish accent. Report $E_{1}$ says that $\mathrm{Mr} \mathrm{X}$ asked for information with a palpable French accent. Report $E_{2}$ says that $\mathrm{Mr} \mathrm{X}$ was chatting with a distinctive German inflection. Finally, report $E_{3}$ says that $\mathrm{Mr} \mathrm{X}$ was arguing with a clear Italian pronunciation. Each of the reports confirms $H$. Yet the conjunction of all of them, though perhaps corroborates the claim $\mathrm{Mr} \mathrm{X}$ is a smart impostor or a fine actor, does not confirm $H$. This will plausibly be the detective's conclusion.

In the above example, the set of all reports appears strongly incoherent (given background knowledge about second language competence). It seems however intuitive that

\footnotetext{
${ }^{24}$ This is also a version of an example originally given in Salmon 1973: 80 .
} 
if the reports received after $E$ had cohered sufficiently with $E$, the conjunction of all reports would have confirmed $H$. (CEG) provides a formal rationale for intuitions of this type. Consider an alternative scenario in which the same detective receives, after $E$, the following reports: report $E_{4}$ says that $\mathrm{Mr} \mathrm{X}$ bought a Spanish newspaper. Report $E_{5}$ says that $\mathrm{Mr} \mathrm{X}$ had dinner in a Spanish restaurant several times. Report $E_{6}$ says that $\mathrm{Mr} \mathrm{X}$ asserted in conversation that he has Spanish relatives. Given that $E$ confirms $H$ and the three additional reports appear to cohere very much with $E$, it seems plausible to conclude that the conjunction $E \wedge E_{4} \wedge E_{5} \wedge E_{6}$ confirms $H$ too. Suppose then that $\left\{E, E_{4}, E_{5}, E_{6}\right\} \in \mathbf{S}$ and that our coherence measure $C$ satisfies (CEG). If the probability function $P$ is defined such that $P(E \wedge$ $\left.E_{4} \wedge E_{5} \wedge E_{6}\right)>0$ and $C\left(\left\{E, E_{4}, E_{5}, E_{6}\right\}\right) \geq c_{E, H}, E \wedge E_{4} \wedge E_{5} \wedge E_{6}$ must necessarily confirm $H$. Importantly, (CEG) can vindicate the use of coherence to make confirmation process comply with the total evidence condition. The latter is a methodological constraint accepted by many epistemologists that requires that the confirmation of a hypothesis be estimated on the grounds of all evidence available to a rational agent in a given time. ${ }^{25}$ Consider for example a scientist who is to assess a hypothesis $H$. Initially, $H$ appears only weakly plausible to him, given background knowledge. Yet, in a given time, the scientist obtains a report $E$ that confirms $H$. Very plausibly, $E$ does not encompass all evidence available to the scientist in that time. Suppose that his total evidence comes in the terms of a finite conjunction of statements $E \wedge E_{1} \wedge \ldots \wedge E_{n}$. Complying with the total evidence conditions requires ascertaining whether $E \wedge E_{1} \wedge \ldots \wedge E_{n}$ confirms $H$. The answer may be difficult: for instance, if none of the conjuncts $E_{i}$ is a falsifier of $H$, total evidence can confirm $H$, but it

\footnotetext{
${ }^{25}$ This constraint appears justified. Consider a scientist who decides to keep working on a hypothesis $H$, or to prefer $H$ to a rival $H^{*}$, on the grounds of confirmation given evidence $E$. If the $E$ were just a part of the total evidence available to the scientist, his decisions could be argued not to be fully rational. For considering all evidence available may for instance reveal that $H$ is in fact at odds with experimental results or that $H^{*}$ is more supported by observations.
} 
may not be obvious whether it does. A way to answer this question is verifying whether the coherence of $E, E_{1}, \ldots, E_{n}$ is sufficiently high on background information. ${ }^{26}$ If this is the case, it is intuitively plausible that total evidence confirms $H$. When coherence is measured by a function that satisfies (CEG), once more, this intuitive confirmation procedure has a formal rationale.

\section{Conclusion}

Recent works in epistemology have challenged the thesis that coherence is truth conducive in the sense that more coherent sets are always more probable. I have argued that should this thesis prove actually false, it would not follow that coherence is a useless notion in epistemology and in philosophy of science. For coherence is confirmation conducive. I have given examples that illustrate how coherent sets of statements facilitate the confirmation of their members by external statements, and the confirmation of external statements by their members. I have argued that the different ways in which coherence appears confirmation conducive can be framed into well-defined properties - precisely, the two confirmation transmission properties (CTC) and (CT), defined in Dietrich and Moretti 2005, and the two evidence gathering properties (CEG) and (EG), characterized in this paper. Olsson's coherence measure does satisfy each of these four properties. Fitelson's coherence measure satisfies a weaker version of (CT) and a weaker version of (EG). I have shown that these properties can provide formal explication of inferences and confirmation procedures typical of everyday and scientific reasoning. Other interesting measures yet to be specified might also satisfy (CTC) and (CT) (and so (CEG) and (EG)) or properties that come close to them. This would open the notion of confirmation conduciveness of coherence to a wider range of coherence measures.

\footnotetext{
${ }^{26}$ Whether background knowledge should be included in total evidence is a matter of convention.
} 


\section{Acknowledgments}

I am very grateful to Franz Dietrich and Iñaki San Pedro Garcia for important comments and criticism upon previous versions of this paper.

\section{References}

Bovens, L and S. Hartmann 2003. Bayesian Epistemology. Oxford University Press. Oxford

Dietrich, F and L. Moretti. 2005. "On coherent sets and the transmission of confirmation". Philosophy of Science 72(3): 403-24.

Fitelson, B. 2004. 'Two technical corrections to my coherence measure'. Paper presented at the Bayesian Epistemology Conference, London School of Economics and Political Science.

Fitelson, B. 2003. 'A Probabilistic Theory of Coherence'. Analysis 63: 194-9.

Kemeny, J. and Oppenheim. 1952. 'Degrees of Factual Support'. Philosophy of Science 19: $307-24$.

Laudan, L. and J. Leplin 1991. 'Empirical Equivalence and Underdetermination'. Journal of Philosophy 88: 449-72.

Okasha, S. 1997. 'Laudan and Leplin on Empirical Equivalence'. The British Journal for Philosophy of Science 48: 251-6.

Olsson, E. 2005. Against Coherence: Truth, Probability, and Justification. Oxford University Press. Oxford.

Olsson, E. 2002. 'What is the problem of coherence and truth?'. Journal of Philosophy 94: 246-72.

Salmon, W. C. 1973. 'Confirmation'. Scientific American 228: 75-84.

Shogenji, T. 1999. 'Is coherence truth-conductive?'. Analysis 59: 339-45. 